\title{
REVIEW
}

\section{Can 'permissive' hypercapnia modulate the severity of sepsis-induced ALI/ARDS?}

\author{
Gerard Curley, Mairead Hayes, and John G Laffey* \\ This article is one of eleven reviews selected from the Annual Update in Intensive Care and Emergency Medicine 2011 (Springer Verlag) and \\ co-published as a series in Critical Care. Other articles in the series can be found online at http://ccforum.com/series/annual. Further \\ information about the Annual Update in Intensive Care and Emergency Medicine is available from http://www.springer.com/series/8901
}

\begin{abstract}
Introduction
Ventilatory strategies that reduce lung stretch by reducing tidal and minute ventilation, which results in a 'permissive' hypercapnic acidosis, improve outcome in patients with acute lung injury/acute respiratory distress syndrome (ALI/ARDS) [1,2]. Reassuringly, evidence from clinical studies attests to the safety and lack of detrimental effects of hypercapnic acidosis [2]. Of particular importance, a secondary analysis of data from the ARDSnet tidal volume study [1] demonstrated that the presence of hypercapnic acidosis at the time of randomization was associated with improved patient survival in patients who received high tidal volume ventilation [3]. These findings have resulted in a shift in paradigms regarding hypercapnia - from avoidance to tolerance with hypercapnia increasingly permitted in order to realize the benefits of low lung stretch. Consequently, low tidal and minute volume ventilation and the accompanying 'permissive' hypercapnia are now the standard of care for patients with ALI/ARDS, and are increasingly used in the ventilatory management of a diverse range of diseases leading to acute severe respiratory failure, including asthma and chronic obstructive pulmonary disease.

The inflammatory response plays a central role in the pathogenesis of injury and in the repair process in ALI/ ARDS [4]. Inflammation is a highly conserved process in evolution, which is essential for survival. It functions to resolve the injurious process, facilitate repair, and return the host to a state of homeostasis. The ideal inflammatory process is rapid, causes focused destruction of pathogens, yet is specific and ultimately self-limiting [5]. In contrast, when the inflammatory response is dysregulated or persistent, this can lead to excessive host damage, and
\end{abstract}

\footnotetext{
*Correspondence: john.laffey@nuigalway.ie

Department of Anestheisa, Clinical Sciences Institute, National University, Galway, Ireland
} translation, reprinting, reuse of illustrations, recitation, broadcasting, reproduction on microfilm or in any other way, and storage in data banks. Duplication of this publication or parts thereof is permitted only under the provisions of the German Copyright Law of September 9 , 1965 , in its current version, and permission for use must always be obtained from Springer-Verlag. Violations are liable for prosecution under the German Copyright Law. contribute to the pathogenesis of lung and systemic organ injury, leading to multiple organ failure and death. The potential for hypercapnia and/or its associated acidosis to potently inhibit the immune response is increasingly recognized [6,7]. Where the host immune response is a major contributor to injury, such as in non-septic ALI/ ARDS, these effects would be expected to result in potential benefit. This has been demonstrated clearly in relevant pre-clinical ALI/ARDS models, where hypercapnic acidosis has been demonstrated to attenuate ALI induced by free radicals [8], pulmonary [9] and systemic ischemia-reperfusion [10], pulmonary endotoxin instillation [11], and excessive lung stretch [12]. The protective effects of hypercapnic acidosis in these models appear due, at least in part, to its anti-inflammatory effects.

The effects of hypercapnia in sepsis-induced lung injury, where a robust immune response to microbial infection is central to bacterial clearance and recovery, is less clear. Of concern, severe sepsis-induced organ failure, whether pulmonary or systemic in origin, is the leading cause of death in critically ill adults and children [13]. Sepsis-induced ARDS is associated with the highest mortality rates. Evidence suggests that approximately $40 \%$ of patients with severe sepsis develop ARDS [13]. Furthermore, infection frequently complicates critical illness due to other causes, with an infection prevalence of over $44 \%$ reported in this population [14]. These issues underline the importance of understanding the effects of hypercapnia on the immune response, and the implications of these effects in the setting of sepsis.

\section{Hypercapnia and the innate immune response} Function of the innate immune response

The immune system can be viewed as having two interconnected branches, namely the innate and adaptive immune responses [5]. The innate immune system is an ancient, highly conserved response, being present in 
some form in all metazoan organisms. This response is activated by components of the wall of invading microorganisms, such as lipopolysaccharide (LPS) or peptidoglycan, following the binding of these pathogen-associated molecular patterns to pattern recognition receptors, such as the Toll-like receptors (TLRs) on tissue macrophages. The innate immune response is also activated by endogenous 'danger' signals, such as mitochondrial components [15], providing an elegant explanation for why non-septic insults can also lead to organ injury and dysfunction. An inflammatory cascade is then initiated, involving cytokine signaling activation of phagocytes that kill bacteria, as is activation of the (later) adaptive immune response.

\section{Activation of the innate immune response}

Hypercapnic acidosis has been demonstrated to inhibit multiple components of the host innate immune responses. Activation of the innate immune response initiates a conserved signaling cascade that culminates in the activation of transcription factors, such as nuclear factor kappa-B (NF-kB) [5]. These transcription factors drive the expression of multiple genes that activate and regulate the pro-inflammatory and repair processes. Increasing evidence suggests that hypercapnic acidosis directly inhibits the activation of NF-kB [16]. Intriguingly, this effect of hypercapnic acidosis may be a property of the $\mathrm{CO}_{2}$ rather than its associated acidosis [17-19]. If confirmed, this finding suggests the presence of a molecular $\mathrm{CO}_{2}$ sensor in mammalian cells. This mechanism of action of hypercapnic acidosis has been demonstrated to underlie some of the anti-inflammatory effects of hypercapnia [16], and to be a key mechanism by which hypercapnia - whether buffered or not - reduces pulmonary epithelial wound healing [18].

\section{Coordination of the innate immune response}

Hypercapnic acidosis also interferes with coordination of the innate immune response by reducing cytokine signaling between immune effector cells. Hypercapnic acidosis reduces neutrophil [20] and macrophage [21] production of pro-inflammatory cytokines such as tumor necrosis factor (TNF)- $\alpha$, interleukin (IL)-1 $\beta$, IL-8 and IL-6. Hypercapnic acidosis reduced endotoxin stimulated macrophage release of TNF $\alpha$ and IL-1 $\beta$ in vitro [21]. Peritoneal macrophages incubated under hypercapnic conditions demonstrated a prolonged reduction in endotoxinstimulated TNF- $\alpha$ and IL-1 $\beta$ release [22]. In contrast, a recent study reported rapid onset and rapid reversibility of IL-6 inhibition by hypercapnia in mature macrophage stimulated with LPS [19]. The mechanism underlying hypercapnic acidosis-mediated inhibition of cytokine and chemokine production appears to be mediated at least in part via inhibition of activation of NF- $\mathrm{kB}$.

\section{The cellular innate immune response}

Neutrophils and macrophages are important effectors of the innate immune response in the setting of bacterial infection. Neutrophils rapidly migrate from the bloodstream to areas of infection, and rapidly phagocytose invading microorganisms. Tissue macrophages and their blood borne monocyte counterparts are activated by bacterial products such as endotoxin, and coordinate the activation of the adaptive immune response in the setting of infection by presenting foreign antigen to lymphocytes and secreting chemokines. Both monocytes and macrophages phagocytose and kill pathogens by similar mechanisms but at a slower rate than neutrophils.

Hypercapnic acidosis may impact on the cellular immune response via both direct and indirect mechanisms. Hypercapnic acidosis inhibits neutrophil expression of the chemokines, selectins and intercellular adhesion molecules $[16,20]$, which facilitate neutrophil binding to the endothelium and migration out of the vascular system. The potential for hypercapnic acidosis to inhibit neutrophil chemotaxis and migration to the site of injury has been confirmed in vivo, where hypercapnic acidosis inhibits pulmonary neutrophil infiltration in response to endotoxin instillation [11]. Hypercapnic acidosis directly impairs neutrophil phagocytosis in vitro [23]. This inhibitory effect appears to be a function of the acidosis per se, with buffering restoring neutrophil phagocytosis [24]. Hypercapnic acidosis also inhibits phagocytosis of opsonized polystyrene beads by human alveolar macrophages, although the levels of $\mathrm{CO}_{2}$ utilized to demonstrate this effect were well beyond the range encountered clinically [19].

Neutrophils and macrophages kill ingested bacteria by producing free radicals such as superoxide, hydrogen peroxide, and hypochlorous acid, and releasing these into the phagosome. This is a $\mathrm{pH}$-dependent process, with free radical production decreased at low $\mathrm{pH}$ [25]. Hypercapnic acidosis inhibits the generation of oxidants such as superoxide by unstimulated neutrophils and by neutrophils stimulated with opsonized Escherichia coli or with phorbol esters [20]. In contrast, hypocapnic alkalosis stimulates neutrophil oxidant generation [20]. Inhibition of the intracellular $\mathrm{pH}$ changes with acetazolamide attenuated these effects. More recently, hypercapnic acidosis has been demonstrated to reduce oxidative reactions in the endotoxin injured lung by a mechanism involving inhibition of myeloperoxidase-dependent oxidation [26]. The potential for hypercapnic acidosis to reduce free radical formation, while beneficial where host oxidative injury is a major component of the injury process, may be disadvantageous in sepsis, where free radicals are necessary to cause bacterial injury and death.

Neutrophil apoptosis following phagocytic activity generally occurs within 48 hours of release into the 
circulation. Conversely, neutrophil death via necrosis causes release of intracellular contents, including harmful enzymes, which can cause tissue destruction. Neutrophils appear to have an increased probability of dying by necrosis following intracellular acidification during phagocytosis [27]. Hypercapnic acidosis may, therefore, increase the probability of neutrophil cell death occurring via necrosis rather than apoptosis.

\section{Hypercapnia and the adaptive immune response}

The adaptive immune system is activated by the innate response following activation of pattern recognition receptors that detect molecular signatures from microbial pathogens. Specific major histocompatibility complex molecules on $\mathrm{T}$ and $\mathrm{B}$ lymphocytes also bind microbial components. These activation events lead to the generation of $\mathrm{T}$ and $\mathrm{B}$ lymphocyte-mediated immune responses over a period of several days.

Much of the focus to date regarding the effects of hypercapnic acidosis on immune response to injury and/ or infection has been on the innate immune response. Less is known about the effects of hypercapnic acidosis on adaptive or acquired immunity. However, important clues as to the potential for hypercapnic acidosis to modulate the adaptive response come from the cancer literature. The tumor microenvironment is characterized by poor vascularization, resulting in tissue hypoxia and acidosis. In a situation analogous to sepsis, acidosis in this setting may hamper the host immune response to tumor cells, potentially leading to increased tumor growth and spread. The cytotoxic activity of human lymphokine activated killer cells and natural killer cells is diminished at acidic $\mathrm{pH}$ [28]. Metabolic acidosis reduces lysis of various tumor cell lines by cytotoxic T-lymphocytes [29]. In contrast, the motility of IL-2-stimulated lymphocytes appears to be stimulated in the presence of an acidified extracellular matrix and severe extracellular acidosis $(\mathrm{pH}$ 6.5) also appears to enhance the antigen presenting capacity of dendritic cells [30]. The net effect of these contrasting actions of metabolic acidosis on the adaptive immune response is unclear. However, the demonstration that hypercapnic acidosis enhanced systemic tumor spread in a murine model [31] raises clear concerns regarding the potential for hypercapnic acidosis to suppress cell-mediated immunity.

\section{Hypercapnia and acidosis modulate bacterial proliferation}

Carbon dioxide has broadly similar effects within the various families of microorganisms, but the sensitivity to $\mathrm{CO}_{2}$ varies across the families, e.g., yeasts are quite resistant to the inhibitory effects of $\mathrm{CO}_{2}$, Gram-positive organisms are somewhat less resistant, and Gramnegative organisms are the most vulnerable [32]. Optimal anaerobic $E$. coli growth occurs at a $\mathrm{CO}_{2}$ tension $\left(\mathrm{PCO}_{2}\right)$ of 0.05 atmospheres, which is similar to the $\mathrm{PCO}_{2}$ in the gut. The aerobic growth rate of $E$. coli was not inhibited by a $\mathrm{PCO}_{2}$ of 0.2 atmospheres but was inhibited at partial pressures above 0.6 atmospheres [33]. It is important to remember that these levels are extremely high in the context of human physiology.

Of concern, however, is the demonstration by Pugin et al. that more clinically relevant degrees of metabolic acidosis can directly enhance bacterial proliferation in vitro [34]. Cultured lung epithelial cells exposed to cyclic stretch similar to that seen with mechanical ventilation produced a lactic acidosis that markedly enhanced the growth of $E$. coli [34]. This was a direct effect of hydrogen ions, as direct acidification of the culture medium to a $\mathrm{pH}$ of 7.2 with hydrochloric acid enhanced $E$. coli growth. In contrast, alkalinizing the $\mathrm{pH}$ of conditioned media from stretched lung cells abolished the enhancement of E. coli growth. A range of Gram-positive and Gramnegative bacteria (including E. coli, Proteus mirabilis, Serratia rubidaea, Klebsiella pneumoniae, Enterococcus faecalis, and Pseudomonas aeruginosa) isolated from patients with ventilator-associated pneumonia (VAP), grew better in acidified media (Fig. 1). Interestingly, this effect was not seen with a methicillin resistant Staphylococcus aureus (MRSA) strain, which appeared to grow best at an alkaline $\mathrm{pH}$ [34].

The effects of hypercapnic acidosis on bacterial proliferation at levels encountered in the context of permissive hypercapnia are unclear. The net effect is likely to be a combination of the effects of the acidosis and of the hypercapnia. Nevertheless, the demonstration that clinically relevant levels of metabolic acidosis enhance bacterial growth is of concern.

\section{Implications for hypercapnia in sepsis}

Immunocompetence is essential to an effective host response to microbial infection. Hypercapnia and/or acidosis may modulate the interaction between host and bacterial pathogen via several mechanisms, resulting in a broad based suppression of the inflammatory response.

\section{Hypercapnia, acidosis and the host response}

The initial host response to invading pathogens is dominated by neutrophil activation, migration to the infective site, and phagocytosis and killing of bacteria. Compartmentalized release of neutrophil proteolytic enzymes and myeloperoxidase-dependent oxygen radicals results in effective pathogen destruction. However, excessive release of these potent mediators into the extracellular space results in damage to host tissue and worsening ALI. Consistent with this is the finding that recovery of neutrophil count in neutropenic patients worsens the severity of ALI [35]. Hypercapnic acidosis may reduce 

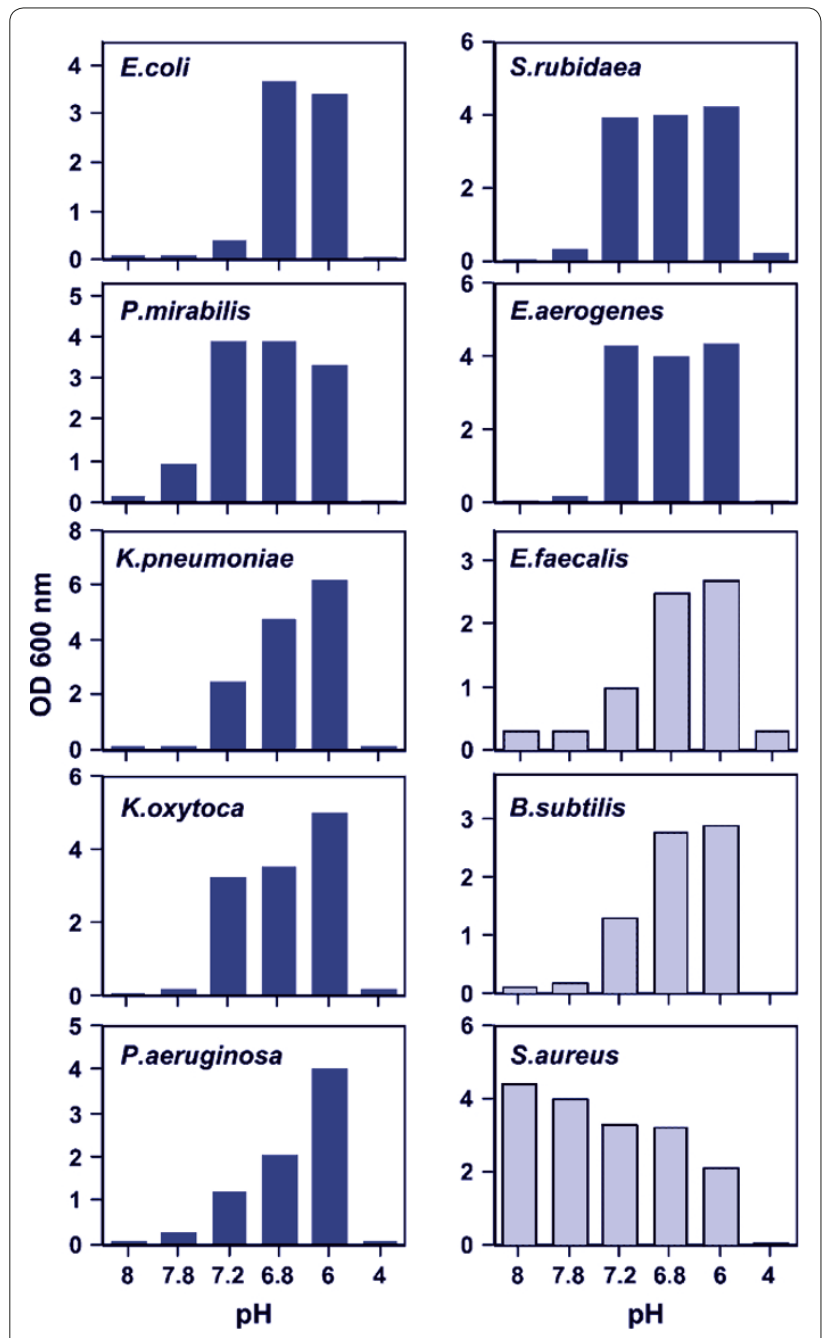

Figure 1. Bacterial pathogens proliferate more rapidly in the setting of metabolic acidosis. All bacterial strains tested, except for a methicillin-resistant $S$. aureus, had a marked growth advantage at moderately acidic pH levels (7.2-7.6) relevant to the clinical setting. Gram-negative bacteria are represented by dark blue bars while Gram-positive bacteria are represented by light blue bars. From [34] with permission.

the potential for damage to host tissue during the response to infection, by reducing lung neutrophil recruitment [10], adherence [16], intracellular $\mathrm{pH}$ regulation [12], oxidant generation [8], and phagocytosis [23]. These mechanisms are considered to underlie some of the protective effects of hypercapnic acidosis in nonsepsis induced ALI [7]. However, these effects of hypercapnic acidosis may be detrimental in sepsis, given the central role of neutrophil mediated phagocytosis of microbial pathogens and activation of the cytokine cascade to the host response to infection. In this context, defects in neutrophil function are associated with increased sepsis severity and worse outcome [36].
Early versus late bacterial infection

The effects of this hypercapnic acidosis-induced immune modulation may vary depending upon the stage of the infective process. The anti-inflammatory properties of hypercapnic acidosis may reduce the intensity of the initial host response to infection, thus attenuating tissue damage (Fig. 2). However, the mechanisms whereby bacteria mediate tissue injury are complex and not limited to the contribution from an excessive host response. In late or prolonged pneumonia, in which tissue injury from direct bacterial spread and invasion makes a significant contribution, hypercapnic acidosis might impair bactericidal host responses. In the absence of effective antibiotic therapy, this may lead to enhanced bacterial spread and replication leading to more severe tissue destruction and lung and systemic organ injury (Fig. 2).

\section{Impact on repair following injury}

Hypercapnic acidosis has been demonstrated to retard the repair process following lung cell and tissue injury. Hypercapnia slowed resealing of stretch-induced cell membrane injuries [37] and inhibited the repair of pulmonary epithelial wounds [18] by a mechanism involving inhibition of the NF-kB pathway. These findings raise the potential that hypercapnic acidosis could lead to increased bacterial translocation through defects in the pulmonary epithelium, while also delaying the recovery process following a septic insult.

Recent studies in relevant preclinical models have significantly advanced our understanding of the effects of hypercapnic acidosis in both pulmonary and systemic sepsis-induced ALI/ARDS. These studies reveal the importance of severity, site, and stage of the infective process, the need for antibiotic therapy, and the utility of buffering the hypercapnic acidosis in this setting.

\section{Hypercapnia in pulmonary sepsis Early lung infection}

The effect of hypercapnic acidosis on pneumonia-induced ALI appears to depend on the stage and severity of the infection. In an acute severe bacterial pneumoniainduced lung injury, hypercapnic acidosis improved physiological indices of injury [38]. Intriguingly, these protective effects were mediated by a mechanism independent of neutrophil function. In contrast, hypercapnic acidosis did not alter the magnitude of lung injury in a less severe acute bacterial pneumonia [39]. Importantly these in vivo studies showed no increase in bacterial count in animals exposed to hypercapnic acidosis, a reassuring finding given concerns regarding retardation of the host bactericidal response and potential bacterial proliferation.

In the clinical setting, many critically ill patients will have established infection at the time of presentation. 


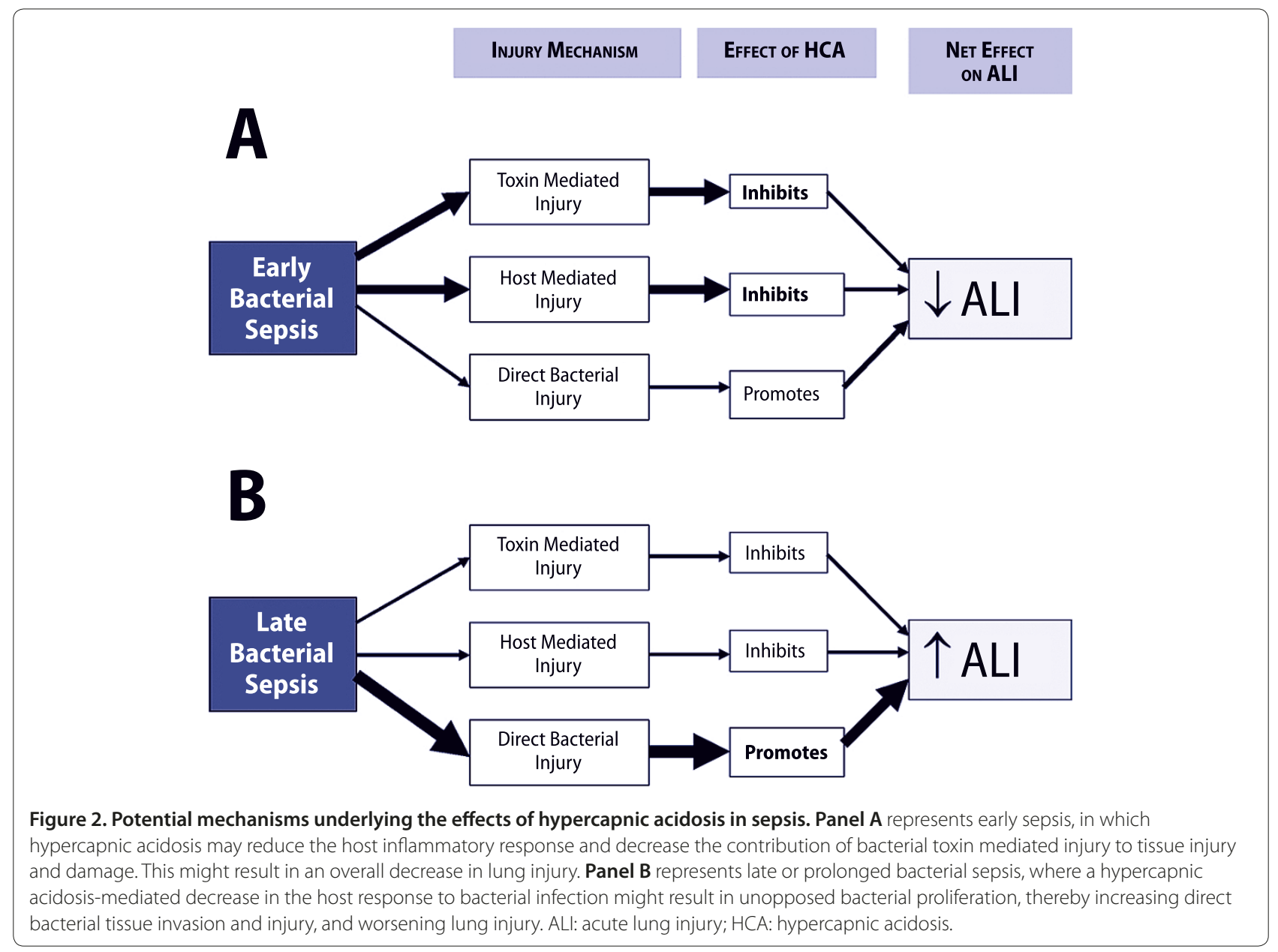

Thus animal models of established bacterial pneumonia, in which hypercapnic acidosis was introduced several hours following induction of infection with $E$. coli, more closely resemble the clinical setting. In an established pneumonia model, hypercapnic acidosis induced after the development of a significant pneumonia-induced lung injury reduced physiological indices of lung injury [40]. Of importance, these protective effects of hypercapnic acidosis were enhanced in the presence of appropriate antibiotic therapy [40]. Again, reassuringly, lung bacterial loads were similar in the hypercapnic acidosis and normocapnia groups [40].

\section{Prolonged lung infection}

In an animal model of prolonged untreated pneumonia, sustained hypercapnic acidosis worsened histological and physiological indices of lung injury, including compliance, arterial oxygenation, alveolar wall swelling and neutrophil infiltration [23]. Of particular concern to the clinical setting, hypercapnic acidosis was associated with a higher lung bacterial count. The mechanism underlying this effect appeared to be inhibition of neutrophil function, as evidenced by impaired phagocytotic ability in neutrophils isolated from hypercapnic rats [23]. Of importance to the clinical context, the use of appropriate antibiotic therapy abolished these deleterious effects of hypercapnia, reducing lung damage and lung bacterial load to levels comparable to those seen with normocapnia.

These findings have been confirmed and considerably expanded in a recent study of hypercapnia in the fruit fly [41]. Helenius et al., in a series of elegant in vivo studies, found that prolonged hypercapnia decreased expression of specific anti-microbial peptides in Drosophilia melanogaster [41]. Hypercapnia decreased bacterial resistance in adult flies exposed to pathogens as evidenced by increased bacterial loads and increased mortality in flies inoculated with E. faecalis, A. tumefaciens, or S. aureus [41]. The previously demonstrated suppressive effects of hypercapnic acidosis on the NF-kB pathway appeared to underlie the decreased resistance to infection [41]. These findings raise significant concerns regarding the safety of hypercapnia in the setting of prolonged pneumonia, particularly in the absence of effective antibiotic therapy. 


\section{Hypercapnia in systemic sepsis}

A growing body of evidence attests to a beneficial role of hypercapnia in the setting of systemic sepsis. Improvements in hemodynamic parameters and lung injury have been demonstrated in evolving, established, and prolonged systemic sepsis in animal models. This is in contrast to the detrimental effects of hypercapnic acidosis seen in prolonged pulmonary sepsis, suggesting that the effects of hypercapnic acidosis depend not only on the stage of the infective process, but also on the site of the primary infection.

\section{Early systemic sepsis}

Hypercapnic acidosis reduces the severity of early septic shock and lung injury induced by systemic sepsis. In a rodent model of peritoneal sepsis induced by cecal ligation and puncture, hypercapnic acidosis slowed the development of hypotension, preserved central venous oxygen saturation, and attenuated the rise in serum lactate compared to control conditions, in the first 3 hours post injury [42]. The severity of early lung injury was reduced as evidenced by a decrease in the alveolararterial oxygen gradient, and reduced lung permeability, compared to normocapnia. Alveolar neutrophil concentration was reduced by hypercapnic acidosis but IL- 6 and TNF- $\alpha$ were unchanged [42]. Of importance, there were no differences in bacterial loads in the lung, blood, or peritoneum in the hypercapnia group.

\section{Prolonged systemic sepsis}

Using an ovine model of fecal peritonitis, Wang et al compared the effects of hypercapnic acidosis with those of dobutamine [43]. Over an 18-hour study period, hypercapnic acidosis resulted in improved hemodynamics of a magnitude comparable to that of dobutamine. Compared with normocapnia, both hypercapnic acidosis and dobutamine raised cardiac index and systemic oxygen delivery and reduced lactate levels. In addition, hypercapnic acidosis attenuated indices of lung injury, including lung edema, alveolar-arterial oxygen partial pressure difference and shunt fraction. Hypercapnic acidosis did not decrease survival time compared to normocapnia in this setting [43]. In a more prolonged systemic sepsis model, Costello et al. demonstrated that sustained hypercapnic acidosis reduced histological indices of lung injury compared with normocapnia in rodents following cecal ligation and puncture [42]. Reassuringly there was no evidence of an increased bacterial load in the lung, blood, or peritoneum of animals exposed to hypercapnia.

\section{Intraperitoneal hypercapnia}

Direct intra-abdominal administration of $\mathrm{CO}_{2}-$ by means of a pneumoperitoneum - reduces the severity of

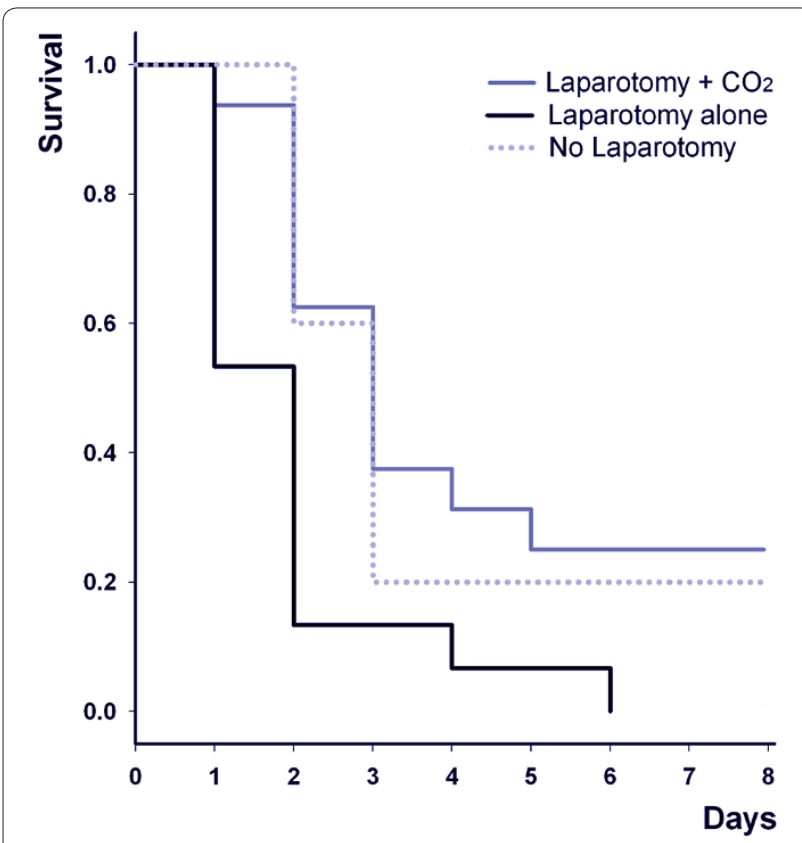

Figure 3. Insufflation of $\mathrm{CO}_{2}$ into the peritoneal cavity improves survival following cecal ligation and puncture-induced systemic sepsis. Animals were first subjected to cecal ligation and puncture. Four hours later, animals underwent a laparotomy and induction of a $\mathrm{CO}_{2}$ pneumoperitoneum (laparotomy $+\mathrm{CO}_{2}$ ), laparotomy alone, or no laparotomy; survival was determined over the following 8 days. Modified from [31] with permission.

abdominal sepsis-induced lung and systemic organ injury. Insufflation of $\mathrm{CO}_{2}$ into the peritoneal cavity prior to laparotomy for endotoxin contamination increased animal survival [44]. Most recently, $\mathrm{CO}_{2}$ pneumoperitoneum has been demonstrated to increase survival in mice with polymicrobial peritonitis induced by cecal ligation and puncture (Fig. 3) [31]. These protective effects of intraperitoneal carbon dioxide insufflation appear be due to the immunomodulatory effects of hypercapnic acidosis, which include an IL-10 mediated downregulation of TNF- $\alpha$ [44]. Importantly, these effects appear to be mediated by the localized peritoneal acidosis, rather than by any systemic effect.

\section{Buffering hypercapnic acidosis in sepsis}

The immunomodulatory effects of hypercapnic acidosis in sepsis may occur as a function of either hypercapnia or acidosis. As discussed, evidence suggests that hypercapnic acidosis exerts certain effects via its associated acidosis [24], while other effects appear be a function of the hypercapnia per se [17]. Buffered hypercapnia, i.e., hypercapnia in the presence of normal $\mathrm{pH}$, may be seen in ALI/ARDS patients as a renal compensatory measure, or as a result of the administration of bicarbonate, a common clinical practice in the ICU, and one that was 
permitted in the ARDSnet tidal volume study [1]. Aside from well established concerns regarding the use of sodium bicarbonate, there is evidence from animal models of lung and systemic sepsis that the anti-inflammatory and protective effects of hypercapnic acidosis are lost with buffering. This has significant implications in clinical scenarios where the buffering of hypercapnia resulting from protective ventilator strategies is considered.

\section{Pulmonary sepsis}

In rodent models of acute pneumonia induced by intratracheal E. coli and by endotoxin, buffered hypercapnia worsened lung injury [24]. Compared with normocapnic controls, buffered hypercapnia increased multiple indices of lung injury including arterial oxygenation, lung compliance, pro-inflammatory pulmonary cytokine concentrations, and measurements of structural lung damage. In these experiments, buffered hypercapnia was established in the animals by exposure to hypercapnic conditions until renal buffering to normal $\mathrm{pH}$ had occurred, thus avoiding the confounding effects of exogenous acid or alkali administration. This contrasts with the protective effects of hypercapnic acidosis in similar models [11,38]. Of note, buffered hypercapnia did not reduce the phagocytic capacity of neutrophils, and did not increase lung bacterial load in these studies [24].

\section{Systemic sepsis}

In a study designed to assess the contribution of acidosis versus hypercapnia to the effects of hypercapnic acidosis on the lung and hemodynamic profile in systemic sepsis, Higgins et al. exposed rats to environmental hypercapnia until renal buffering had restored $\mathrm{pH}$ to the normal range [45]. Both buffered hypercapnia and hypercapnic acidosis reduced the severity of early shock and attenuated the increase in serum lactate compared with normocapnia. In contrast, buffered hypercapnia did not attenuate physiologic or histologic indices of lung injury in these studies [45]. Reassuringly, there was no evidence to suggest that buffered hypercapnia worsened the degree of lung injury compared to normocapnia, and buffered hypercapnia did not increase the bacterial load in the lungs or the bloodstream [45].

\section{Hypercapnia and sepsis: where are we now?}

The generally beneficial effects of hypercapnic acidosis in the setting of experimental non-septic inflammatory injury contrast with a more complex spectrum of effects in the setting of live bacterial infection. Hypercapnia and/ or acidosis exert diverse - and potentially conflicting effects on the innate and adaptive immune responses. Overall, hypercapnic acidosis appears to suppress the immune response, although the net effect of its multiple actions appears to vary depending on the site of infection and also on whether the acidosis produced by the hypercapnia is buffered or not. Hypercapnic acidosis appears to protect the lung from injury induced by evolving or more established lung and systemic bacterial sepsis in relevant pre-clinical models. In contrast, the effects of hypercapnic acidosis in prolonged untreated bacterial sepsis appear to differ depending on the source of the infection, with the immunosuppressive effects of hypercapnic acidosis worsening lung injury in the setting of prolonged pneumonia. This deleterious effect is abrogated by effective antibiotic therapy. In contrast, hypercapnic acidosis reduced lung damage caused by prolonged systemic sepsis, again highlighting the potential importance of the source of infection. Finally, buffering of the acidosis induced by hypercapnia does not confer significant benefit in the setting of lung or systemic sepsis, and may actually worsen lung injury in the setting of pneumonia.

Taken together, recent experimental findings in relevant pre-clinical models provide some reassurance regarding the safety of hypercapnia in sepsis, particularly in early pneumonia, and in the setting of abdominal sepsis. However, in the setting of prolonged pneumonia, the immunosuppressive effects of hypercapnia remain a concern. While the use of ventilation strategies resulting in hypercapnia is clearly justified in patient with ALI/ ARDS, care is warranted in the setting of sepsis. The finding that deleterious effects of hypercapnia in the setting of prolonged pneumonia are abrogated by appropriate antibiotic therapy is of importance.

Clinicians should carefully consider the use of early empiric antibiotic therapy in hypercapnic ALI/ARDS patients in whom sepsis is suspected or confirmed. However, concerns persist, particularly where antibiotic cover may be suboptimal, or the bacteria are more resistant to antibiotic therapy. The findings that hypercapnia may increase septic lung injury in the setting of prolonged pneumonia is also of relevance to other patient groups, such as patients with infective exacerbations of chronic obstructive airways disease.

\section{Conclusion}

Hypercapnia is an integral component of protective lung ventilatory strategies in patients with severe respiratory failure. The potential for hypercapnia to modulate the immune response, and the mechanisms underlying these effects are increasingly well understood. The findings that hypercapnic acidosis is protective in systemic sepsis, and in the earlier phases of pneumonia-induced sepsis, provide reassurance regarding the safety of hypercapnia in the clinical setting. However, the potential for hypercapnic acidosis to worsen injury in the setting of prolonged lung sepsis must be recognized. Additional studies are needed to further elucidate the mechanisms 
underlying the effects of hypercapnia and acidosis in the setting of sepsis-induced lung injury.

\section{Acknowledgement}

This work was supported by funding from the Health Research Board, Dublin, Ireland (Grant No: RP/2008/193), and the European Research Council, Brussels, Belgium, under the Framework 7 Programme (Grant No: ERC-2007-StG 207777).

\section{Competing interests}

The authors declare that they have no competing interests.

\section{List of abbreviations used}

ALI: acute lung injury; ARDS: acute respiratory distress syndrome; IL: interleukin; LPS: lipopolysaccharide; MRSA: methicillin resistant Staphylococcus aureus; NF-kB: nuclear factor kappa-B; TLR: toll-like receptor; TNF: tumor necrosis factor; VAP: ventilator-associated pneumonia.

Published: 22 March 2011

\section{References}

1. The ARDS Network: Ventilation with lower tidal volumes as compared with traditional tidal volumes for acute lung injury and the acute respiratory distress syndrome. N Engl J Med 2000, 342:1301-1308.

2. Hickling KG, Walsh J, Henderson S, Jackson R: Low mortality rate in adult respiratory distress syndrome using low-volume, pressure-limited ventilation with permissive hypercapnia: a prospective study. Crit Care Med 1994, 22:1568-1578.

3. Kregenow DA, Rubenfeld GD, Hudson LD, Swenson ER: Hypercapnic acidosis and mortality in acute lung injury. Crit Care Med 2006, 34:1-7.

4. Ware LB, Matthay MA: The acute respiratory distress syndrome. N Engl J Med 2000, 342:1334-1349.

5. Barton GM: A calculated response: control of inflammation by the innate immune system. J Clin Invest 2008, 118:413-420.

6. Laffey JG, Kavanagh BP: Carbon dioxide and the critically ill-too little of a good thing? Lancet 1999, 354:1283-1286.

7. Laffey JG, O'Croinin D, McLoughlin P, Kavanagh BP: Permissive hypercapniarole in protective lung ventilatory strategies. Intensive Care Med 2004, 30:347-356.

8. Shibata K, Cregg N, Engelberts D, Takeuchi A, Fedorko L, Kavanagh BP: Hypercapnic acidosis may attenuate acute lung injury by inhibition of endogenous xanthine oxidase. Am J Respir Crit Care Med 1998, 158:1578-1584

9. Laffey JG, Jankov RP, Engelberts D, et al:: Effects of therapeutic hypercapnia on mesenteric ischemia-reperfusion injury. Am J Respir Crit Care Med 2003, 168:1383-1390.

10. Laffey JG, Tanaka M, Engelberts D, et al:: Therapeutic hypercapnia reduces pulmonary and systemic injury following in vivo lung reperfusion. Am J Respir Crit Care Med 2000, 162:2287-2294.

11. Laffey JG, Honan D, Hopkins N, Hyvelin JM, Boylan JF, McLoughlin P: Hypercapnic acidosis attenuates endotoxin-induced acute lung injury. Am J Respir Crit Care Med 2004, 169:46-56.

12. Sinclair SE, Kregenow DA, Lamm WJ, Starr IR, Chi EY, Hlastala MP: Hypercapnic acidosis is protective in an in vivo model of ventilator-induced lung injury. Am J Respir Crit Care Med 2002, 166: 403-408.

13. Hudson LD, Milberg JA, Anardi D, Maunder RJ: Clinical risks for development of the acute respiratory distress syndrome. Am J Respir Crit Care Med 1995, 51:293-301

14. Vincent $J$, Bihari DJ, Suter PM, et al:: The prevalence of nosocomial infection in intensive care units in Europe. Results of the European Prevalence of Infection in Intensive Care (EPIC) Study. EPIC International Advisory Committee. JAMA 1995, 274:639-644.

15. Zhang $Q$, Raoof M, Chen Y, et al:: Circulating mitochondrial DAMPs cause inflammatory responses to injury. Nature 2010, 464:104-107.

16. Takeshita K, Suzuki Y, Nishio K, et al.: Hypercapnic acidosis attenuates endotoxin-induced nuclear factor-kappa B activation. Am J Respir Cell Mol Biol 2003, 29:124-132.

17. Cummins EP, Oliver KM, Lenihan CR, et al:: NF-kB links CO2 sensing to innate immunity and inflammation in mammalian cells. J Immunol 2010, 185:4439-44.
18. O'Toole D, Hassett P, Contreras M, et al:: Hypercapnic acidosis attenuates pulmonary epithelial wound repair by an NF-kappaB dependent mechanism. Thorax 2009, 64:976-982.

19. Wang N, Gates KL, Trejo H, et al:: Elevated $\mathrm{CO} 2$ selectively inhibits interleukin- 6 and tumor necrosis factor expression and decreases phagocytosis in the macrophage. Faseb J 2010, 24:2178-2190.

20. Coakley RJ, Taggart C, Greene C, McElvaney NG, O'Neill SJ: Ambient pCO2 modulates intracellular $\mathrm{pH}$, intracellular oxidant generation, and interleukin-8 secretion in human neutrophils. J Leukoc Biol 2002, 71:603-610

21. West MA, Baker J, Bellingham J: Kinetics of decreased LPS-stimulated cytokine release by macrophages exposed to CO2. J Surg Res 1996, 63:269-274.

22. Gupta A, Watson Dl: Effect of laparoscopy on immune function. Br J Surg 2001, 88:1296-1306

23. O'Croinin DF, Nichol AD, Hopkins N, et al:: Sustained hypercapnic acidosis during pulmonary infection increases bacterial load and worsens lung injury. Crit Care Med 2008, 36:2128-2135.

24. Nichol AD, O'Cronin DF, Howell K, et al:: Infection-induced lung injury is worsened after renal buffering of hypercapnic acidosis. Crit Care Med 2009, 37:2953-2961.

25. Swallow CJ, Grinstein S, Sudsbury RA, Rotstein OD: Relative roles of $\mathrm{Na}+/ \mathrm{H}+$ exchange and vacuolar-type $\mathrm{H}+$ ATPases in regulating cytoplasmic $\mathrm{pH}$ and function in murine peritoneal macrophages. J Cell Physio/ 1993, 157:453-460.

26. Nichol AD, O'Cronin DF, Naughton F, Hopkins N, Boylan J, McLoughlin P. Hypercapnic acidosis reduces oxidative reactions in endotoxin-induced lung injury. Anesthesiology 2010, 113:116-125.

27. Coakley RJ, Taggart C, McElvaney NG, O'Neill SJ: Cytosolic pH and the inflammatory microenvironment modulate cell death in human neutrophils after phagocytosis. Blood 2002, 100:3383-3391.

28. Severin T, Muller B, Giese G, et al.: pH-dependent LAK cell cytotoxicity. Tumour Biol 1994, 15:304-310.

29. Redegeld F, Filippini A, Sitkovsky M: Comparative studies of the cytotoxic T lymphocyte-mediated cytotoxicity and of extracellular ATP-induced cell lysis. Different requirements in extracellular Mg2+ and pH. J Immuno/ 1991, 147:3638-3645.

30. Vermeulen M, Giordano M, Trevani AS, et al:: Acidosis improves uptake of antigens and $\mathrm{MHC}$ class I-restricted presentation by dendritic cells. $\mathrm{J}$ Immunol 2004, 172:3196-3204.

31. Metzelder M, Kuebler JF, Shimotakahara A, Chang DH, Vieten G, Ure BL: CO2 pneumoperitoneum increases survival in mice with polymicrobial peritonitis. Eur J Pediatr Surg 2008, 18:171-175.

32. Dixon NM, Kell DB: The inhibition by $\mathrm{CO} 2$ of the growth and metabolism of micro-organisms. J Appl Bacterio/ 1989, 67:109-136.

33. Mori H, Kobayashi T, Shimizu S: Effect of carbon dioxide on growth of microorganisms in fed-batch cultures. J Ferment Technol 1983, 61:211-213.

34. Pugin J, Dunn-Siegrist I, Dufour J, Tissieres P, Charles PE, Comte R: Cyclic stretch of human lung cells induces an acidification and promotes bacterial growth. Am J Respir Cell Mol Biol 2008, 38:362-370.

35. Azoulay E, Darmon M, Delclaux C, et al:: Deterioration of previous acute lung injury during neutropenia recovery. Crit Care Med 2002, 30: 781-786.

36. Alves-Filho JC, de Freitas A, Spiller F, Souto FO, Cunha FQ: The role of neutrophils in severe sepsis. Shock 2008, 30 (Suppl 1): 3-9.

37. Doerr $\mathrm{CH}$, Gajic $\mathrm{O}$, Berrios JC, et al:: Hypercapnic acidosis impairs plasma membrane wound resealing in ventilator-injured lungs. Am J Respir Crit Care Med 2005, 171:1371-1377.

38. Ni Chonghaile M, Higgins BD, Costello JF, Laffey JG: Hypercapnic acidosis attenuates severe acute bacterial pneumonia-induced lung injury by a neutrophil-independent mechanism. Crit Care Med 2008, 36:3135-3144.

39. O'Croinin DF, Hopkins NO, Moore MM, Boylan JF, McLoughlin P, Laffey JG: Hypercapnic acidosis does not modulate the severity of bacterial pneumonia-induced lung injury. Crit Care Med 2005, 33:2606-2612.

40. Chonghaile MN, Higgins BD, Costello J, Laffey JG: Hypercapnic acidosis attenuates lung injury induced by established bacterial pneumonia. Anesthesiology 2008, 109:837-848.

41. Helenius IT, Krupinski T, Turnbull DW, et al.: Elevated CO2 suppresses specific Drosophila innate immune responses and resistance to bacterial infection. Proceedings of the National Academy of Sciences of the United States of America 2009, 106:18710-18715.

42. Costello J, Higgins B, Contreras M, et al:: Hypercapnic acidosis attenuates 
shock and lung injury in early and prolonged systemic sepsis. Crit Care Med 2009, 37:2412-2420.

43. Wang Z, Su F, Bruhn A, Yang X, Vincent JL: Acute hypercapnia improves indices of tissue oxygenation more than dobutamine in septic shock. Am J Respir Crit Care Med 2008, 177:178-183.

44. Fuentes JM, Hanly EJ, Aurora AR, et al:: CO2 abdominal insufflation pretreatment increases survival after a lipopolysaccharide-contaminated laparotomy. J Gastrointest Surg 2006, 10:32-38.

45. Higgins BD, Costello J, Contreras M, Hassett P, D OT, Laffey JG: Differential
Effects of Buffered Hypercapnia versus Hypercapnic Acidosis on Shock and Lung Injury Induced by Systemic Sepsis. Anesthesiology 2009,

111:1317-1326.

doi:10.1186/cc9994

Cite this article as: Curley G, et al:: Can 'permissive' hypercapnia modulate

the severity of sepsis-induced ALI/ARDS?. Critical Care 2011, 15:212. 